\title{
Measuring the Deformation of a Flat Die by Applying a Laser Beam on a Reflecting Surface
}

\author{
W. Assaad ${ }^{1, a}$, H.J.M. Geijselaers ${ }^{2, b}$ and K.E.Nilsen ${ }^{3, c}$ \\ ${ }^{1}$ Materials Innovation Institute, The Netherlands \\ ${ }^{2}$ Faculty of Engineering Technology, University of Twente, The Netherlands \\ ${ }^{3}$ Boal Beheer B.V, De Lier, The Netherlands \\ aw.assaad@m2i.nl, bh.j.m.geijselaers@ctw.utwente.nl, ck.nilsen@boalgroup.com
}

Keywords: extrusion,deformation,decoupled,die.

\begin{abstract}
The design of extrusion dies depends on the experience of the designer. After the die has been manufactured, it is tested during an extrusion process and machined several times until it works properly. The die is designed by a trial and error method which is expensive interms of time consumption and the amount of scrap. Research is going on to replace the trial pressing with finite element simulations that concentrate on material and tool analysis. In order to validate the tool simulations, an experiment is required for measuring the deformation of the die. Measuring the deformation of the die is faced with two main obstacles: high temperature and little free space. To overcome these obstacles a method is tried, which works by applying a laser beam on a reflecting surface. This cheap method is simple, robust and gives good results. This paper describes measuring the deformation of a flat die used to extrude a single $U$ shape profile. In addition, finite element calculation of the die is performed. Finally, a comparison is performed between experimental and numerical results.
\end{abstract}

\section{Introduction}

In a direct aluminum extrusion process the die is subjected to two types of loads: mechanical and thermal loads. These loads cause the die face to deform in a concave shape. Consequently, the section is extruded non uniformly through the deformed die where it is thinner at the middle and thicker at the edges. Therefore, the dimensions of the extruded section may not be as specified. The die must be corrected in order to extrude a profile with the specified dimensions. Prediction of how the die deforms helps in decreasing the number of trial pressings and die corrections. In fact finite element simulations are used in predicting the deformation of the die. Moreover, these simulations require experiments in order to be validated. Therefore, an experiment is conducted for measuring the deformation of a flat die used in the extrusion of a $U$ shape profile. Measuring the die deformation or the pressure on the die face is a challenging task especially in an industrial extrusion environment. In literature different approaches were applied for measuring the pressure on the die face and deformation of the die. In [1] the pressure distribution on the die face and deformation of the die in the extrusion of 1050 aluminum rod were measured by the use of a semi conductor strain gauge pressure sensor and a laser displacement meter respectively. The measurements were performed on a 400 tons vertical laboratory press. The pressure sensor was inserted in a hole drilled through the die and its holder such that the contact between the sensor and the metal would take place. The measurement of the die deformation was performed by measuring the deflection of the bar attached to the die at a specified position. In [2] cylindrical flat steel capsule which deforms linearly was inserted in the die face. The capsule was connected to a deformation measurement system with a bar inserted in a hole drilled through the tool stack. In [3] a technique was designed for measuring the pressure on the die face with the application of Capacitec capacitive probes. This type of sensors was chosen due to their small size and functionality at temperatures above $400 \mathrm{C}^{\circ}$. In 
[3] and [4] the technique was applied successfully in measuring the pressure on the die face during the extrusion of a rod and thin strip with an experimental vertical extrusion press. But it was not a complete success in [5] in measuring the pressure on the die face in an industrial $U$ shape profile extrusion due to failure of the sensors. Two different ideas were utilized in the above mentioned experiments. First, the deflection of the die is measured by measuring the deflection of a bar connected to the die face. Second, the deformation of the die is measured by sensors integrated in the die. Moreover, a special die is required to be designed and manufactured for mounting the sensors and their connectors to the measurement system. In addition calibration is a demand before measurement.

Since the experiment is carried out on a press owned by an extrusion company, modification to the tool stack is limited. Therefore, the application of the second idea is difficult in this case. Finally, a new approach is deduced from the first idea and followed by applying a laser beam on a reflecting surface. A reflecting surface is mounted on the die face. The experiment is carried out successfully in two rounds which guarantee its repeatability. The experimental results of the first round are compared with finite element simulations where a decoupled analysis is applied. The finite element simulations are performed by the in-house implicit FE-program DiekA.

\section{Experiment setup}

The measurement is done on the deformation of a flat die used in producing a single $\mathrm{U}$ shape profile shown in Fig. 1. To avoid interrupting the beam during the experiment, the saw and the stretcher are switched off. The die, backer and ring are mounted together as shown in Fig. 2. Fig.3 illustrates the experiment setup, where a laser beam is emitted from a laser source towards a reflecting surface which reflects the beam on a white screen. The laser source is placed out side the run-out table and far from the press because it works at room temperature.
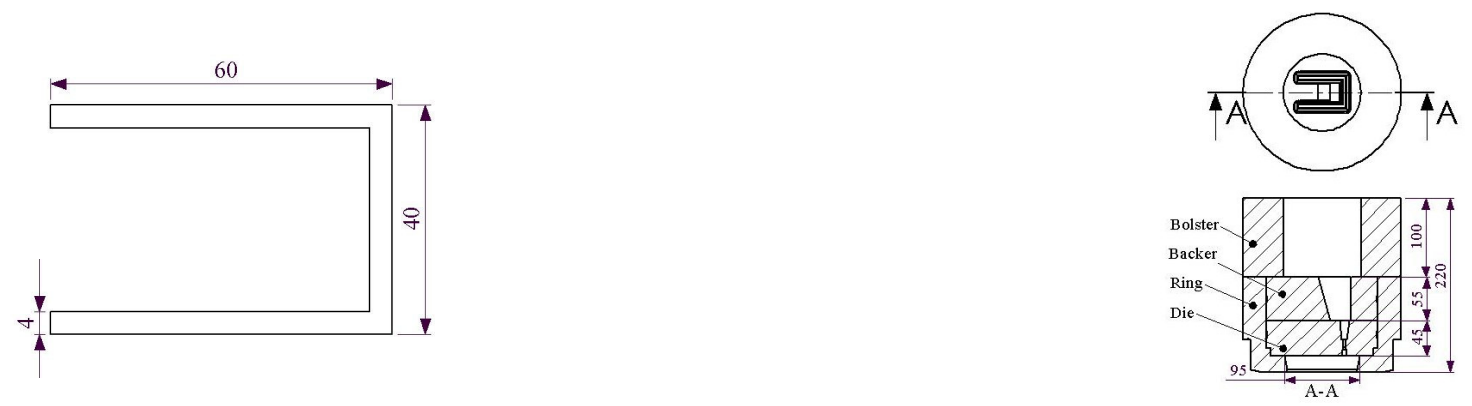

Fig.1: profile (Dimensions in $\mathrm{mm}$ )

Fig.2: tool (Dimensions in $\mathrm{mm}$ )

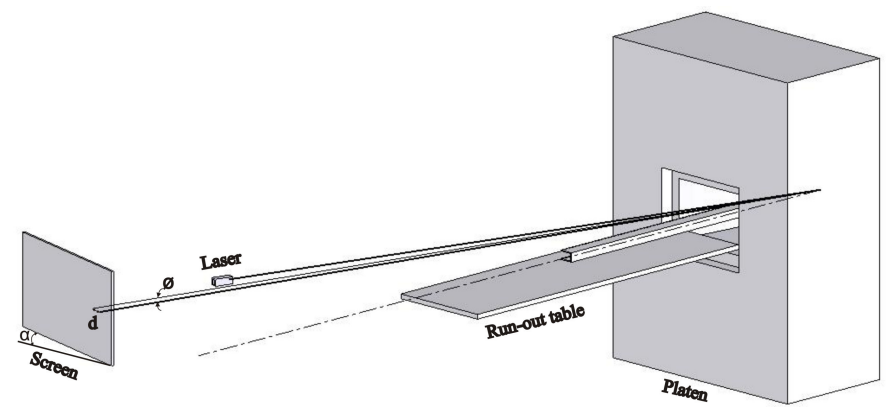

Fig.3: experiment setup

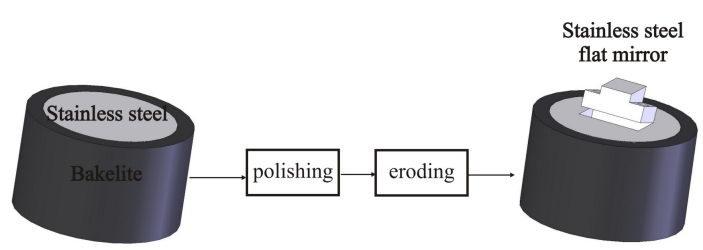

Fig.4: production of the stainless steel flat mirror 
Reflecting surface. A stainless steel flat mirror is attached to the tongue of the die as shown in Fig. 6. Stainless steel is chosen as a reflecting material, because it withstands high temperature and preserves its reflectivity during the experiment. An inclined reflecting surface is designed because the laser source must be placed outside the run-out table. Its angle is determined from the position of the laser source, position of the screen and opening in the pressure ring. The small size and the angle of the reflecting surface are the main obstacles in the polishing stage during its production. Finally, after several trials it was produced in the following manner as shown in Fig. 4: (1) embedding a stainless steel piece in "Bakelite", (2) polishing, (3) cutting the mirror from the middle of the piece by eroding in order to get the best flatness.

Laser source. The laser source is chosen such that the diameter of the spot is less than the length of the side of the reflecting surface. Therefore, a laser source is selected with the following specifications:

1. Green dot laser with $532 \mathrm{~nm}$ wavelength

2. Output power: $20 \mathrm{~mW}$.

3. Divergence: $0.1 \mathrm{mrad}$.

4. Dot diameter varies between $0.4 \mathrm{~mm}$ and $3 \mathrm{~mm}$.

Backer. A new backer displayed in Fig. 5 is produced to accommodate the incident beam, reflected beam and the stainless steel mirror.

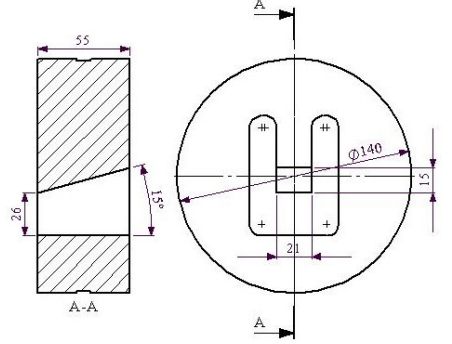

Fig.5: front and section view of the backer [mm]

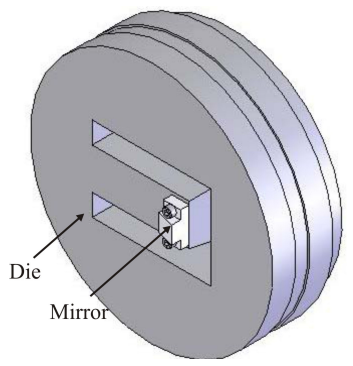

Fig.6: fixation of the mirror

Screen. The reflected beam is projected on a screen. The screen has a white background with four reference points. The reference points are used in calculating the movement of the reflected spot through a bilinear transformation.

Billets. Billets with $92 \mathrm{~mm}$ diameter and 360mm length are extruded. Their material is AA6060 with $0.40 \% \mathrm{Si}$ and $0.45 \% \mathrm{Mg}$.

\section{Experiment procedure}

The following points summarize the procedure of the experiment:

1. Fix the mirror on the die with two M3 bolts as shown in Fig. 6.

2. Assemble the tool parts such as die, backer, and ring.

3. Put the tool in the oven and heat it up to $460 \mathrm{C}^{\circ}$.

4. Place the laser source and screen.

5. Two cameras are placed in two different positions. Camera " $\mathrm{A}$ " is positioned in front of the control panel, and camera "B" in front of the screen.

6. As soon as the temperature of the tool reaches the desired one, it is removed from the oven and placed in the press.

7. Turn on the laser source, aim it at the mirror and adjust the position of the screen until the reflected spot can be seen on it.

8. Turn on the two video cameras and start pressing

9. The locations of the laser source and screen are measured. 


\section{Experimental results}

The two movies are analyzed and the data are extracted for the first four billets. The ram speed, seal pressure and main cylinder pressure are taken out from movie "A". The seal pressure gives the information about locking up the container to the tool. The cylinder pressure is the pressure applied on the ram to extrude the billet. The cylinder pressure and the seal pressure are plotted in Fig.7. After loading the billet in the container, the seal pressure is increased to 210 bars in order to lock up the container to the tool, and then the cylinder pressure is increased to 50 bars in order to compress the billet until it fills the container which is $95 \mathrm{~mm}$ diameter. Then the cylinder pressure is decreased to zero and the container is moved backward to allow the hot gas to escape from the container. This procedure is known as "Burp". Soon afterward the container is closed again and cylinder pressure is increased to 120 bars and extruding the current billet is started. During extrusion the cylinder pressure decreases slightly exponentially due to friction between the billet and the container as described in [6]. After extruding the current billet, the container is moved backward and part of the oil from the hydraulic circuit is passed to shear off the butt end. As shown in Fig 7, the profile of the cylinder pressure during the extrusion of the first billet is different from that of the successor billets because part of the first billet fills the die and the baffle. The extrusion force is calculated from the cylinder pressure and the diameter of the cylinder and displayed in Fig.8. The extrusion force profiles for the third and fourth billets are higher than that of the second billet because of cooling down. The ram speed with a nominal value of $5.3 \mathrm{~mm} / \mathrm{sec}$ is exhibited in Fig.9. The extruded billet length is calculated by integrating the ram speed in time. Table 1 shows that $20 \%$ of the first billet is lost in filling the die and the baffle.

\begin{tabular}{|c|c|c|c|c|}
\hline Billet & 1 & 2 & 3 & 4 \\
\hline Extruded length $[\mathrm{mm}]$ & 250 & 310 & 310 & 310 \\
\hline
\end{tabular}

Table 1: Extruded billet length
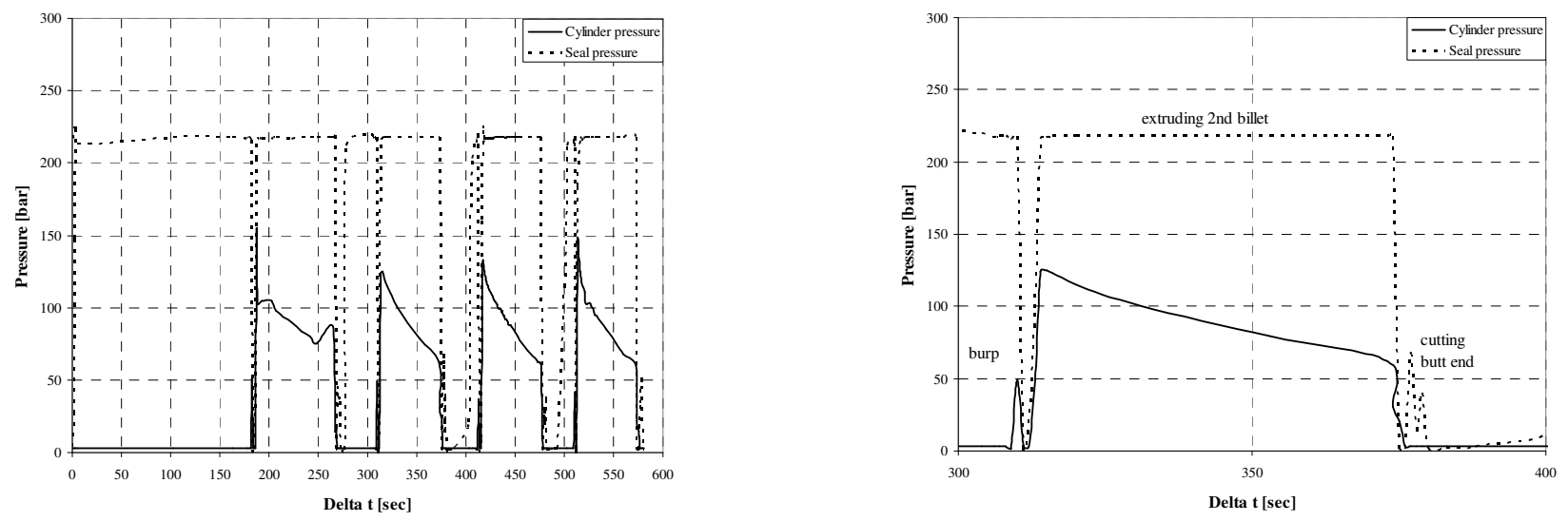

Fig.7: pressure versus time

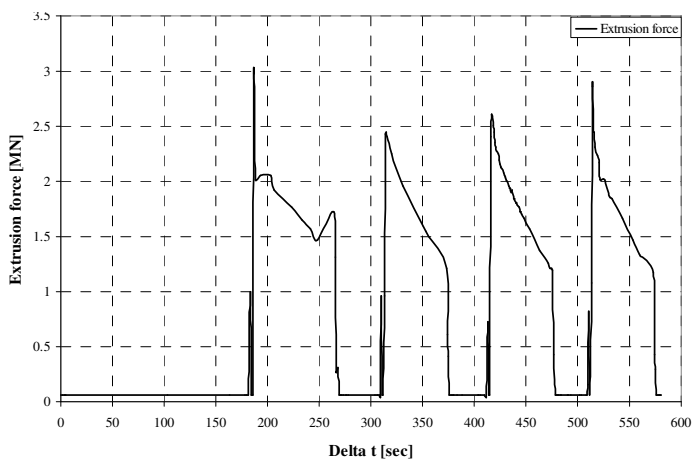

Fig.8: extrusion force versus time

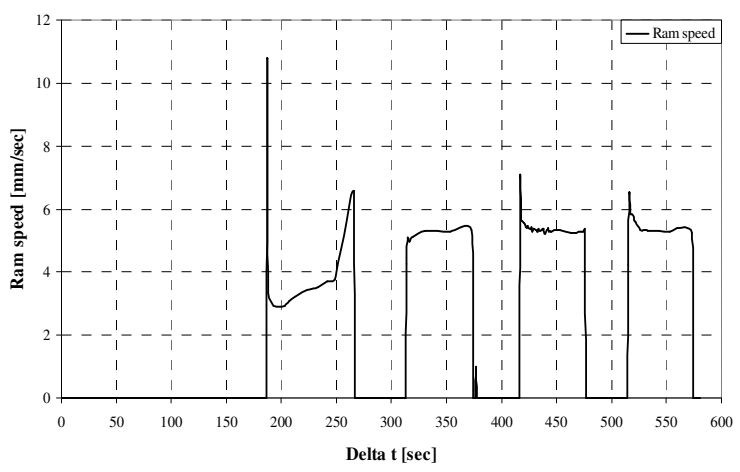

Fig.9: ram speed versus time 
The movement of the reflected spot is determined from movie "B". A program is implemented using image processing toolbox in order to read movie "B" and to calculate the position of the spot during the extrusion process. Moreover, the angular deflection of the tongue is determined from Fig. 10 and equations 1 and 2.

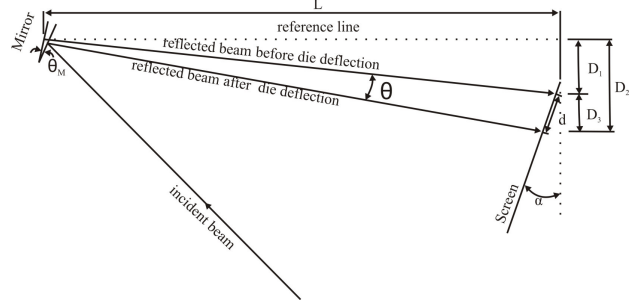

Fig.10: sketch for calculating the angular deflection of the tongue

$$
\begin{aligned}
& \tan \theta=\frac{d * L}{\left(L^{2}+D_{1}^{2}\right) \cos \alpha+\left(D_{1} * d\right)} \\
& \theta_{M}=\theta / 2
\end{aligned}
$$

Where $\theta=$ angular displacement of the reflected beam, $\theta_{M}=$ angular deflection of the tongue, $d=$ total displacement of the spot in $\mathrm{mm}, \mathrm{L}=3376 \mathrm{~mm}, \mathrm{D}_{1}=810 \mathrm{~mm}, \alpha=12$ degrees.

The angular deflection of the tongue is displayed in Fig.11 which shows that it is increasing during the extrusion because the binding force $\left(\mathrm{F}_{\mathrm{b}}\right)$ shown in Fig.12 between the container and the tool decreases with decreasing the friction force between the container and the billet as depicted in [1]. The angular deflection of the tongue reaches a value of $8 \mathrm{mrad}$ and $7 \mathrm{mrad}$ at the end of the extrusion of the first and the successor billets respectively. The flexibility of the die during the extrusion of the first billet leads to higher angular deflection of the tongue. After the first and second rounds of the experiment, the die is checked and $0.03 \mathrm{~mm}$ deviation in the bearing is detected. This amount is equivalent to $0.6 \mathrm{mrad}$ angular deflection of the tongue. The reason of this amount of plastic deformation has not been clarified yet. It may be a production defect or occur during the extrusion of the first billet. Finally a rigid body motion of the tool is detected during shearing off the butt end because it is free.

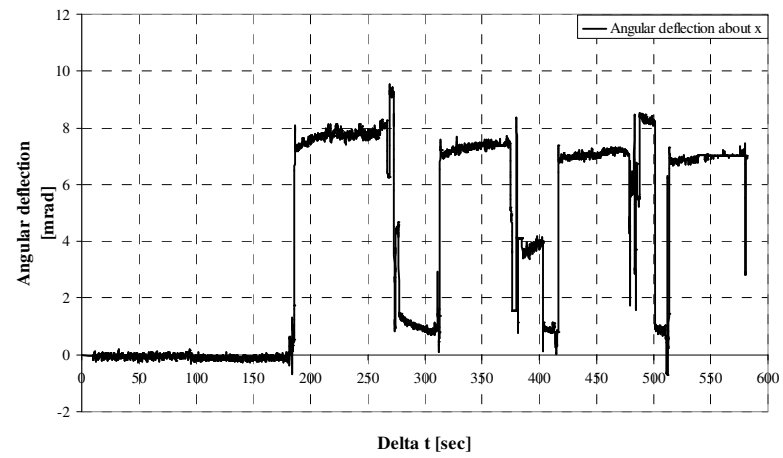

Fig.11: tongue angular deflection versus time

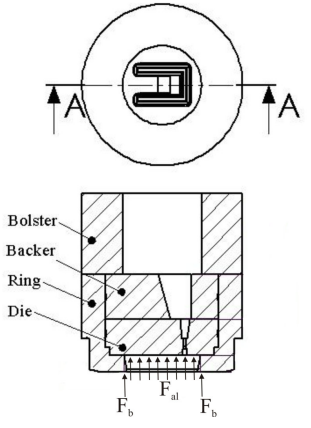

Fig.12: loads on the tool

\section{Numerical analysis}

The decoupled analysis [7] is applied where the billet and the tool are studied separately. First, a 3D isothermal numerical simulation for the billet is performed by applying an Eulerian formulation to over come the problem of large deformation. In fact, a transient calculation which consumes large calculation times is required to evaluate the extrusion pressure at the corresponding ram position. Therefore, a steady state solution of the problem is computed at a specified ram position for avoiding large calculation time. For example in the current simulation, the ram position is defined at 
the end of its stroke which corresponds to $50 \mathrm{~mm}$ of the billet's analyzed length. This length is obtained by the summation of the baffle's length and the butt end length. Moreover, the temperature of the analyzed billet is set similar to the temperature of the extrudate which is about $520 \mathrm{C}^{\circ}$. In addition, a rigid die is assumed where the influence of the die deformation on the material flow is neglected. As soon as the analysis reaches the steady state, the reaction forces in the contact zone between die and billet are calculated and transferred to the die face. Next a 3D isothermal numerical simulation for the tool including die, backer and bolster is performed by applying Updated Lagrangian formulation.

Material models. The behaviour of aluminum alloy (AA6060) is described by von Mises viscoplastic material model. The relation between the flow stress and the equivalent viscoplastic strain rate is described by Sellars-Tegart law in Eq.3 [6]. Since the material constants for (AA6060) are not available and the amounts of $\mathrm{Mg}$ and $\mathrm{Si}$ in the alloy are in the range of that in (AA6063). Then material constants for (AA6063) listed in Table 2 [8] are used.

The tool material (AISIH-13 steel) is described by elasto-plastic material model in which Voce hardening is used to describe the plastic behavior. The material properties at temperatures of $460 \mathrm{C}^{\circ}$ are listed in Table 3 [9].

$\bar{\sigma}=\frac{1}{\alpha} \operatorname{arcsinh}\left(\left(\frac{\dot{\epsilon}}{A} \exp \left(\frac{Q}{R T}\right)\right)^{1 / n}\right)$

\begin{tabular}{|l|l|l|l|l|l|}
\hline $\mathrm{N}$ & $\mathrm{Q}[\mathrm{J} / \mathrm{mol}]$ & $\mathrm{A}\left[\mathrm{s}^{-1}\right]$ & $\mathrm{R}[\mathrm{J} / \mathrm{K} . \mathrm{mol}]$ & $\alpha\left[\mathrm{MPa}^{-1}\right]$ & $\mathrm{T}[\mathrm{K}]$ \\
\hline 5.4 & 140000 & $6.0 \mathrm{E} 09$ & 8.314 & 0.04 & 793 \\
\hline
\end{tabular}

Table 2: Constants used in Sellars-Tegart law

\begin{tabular}{|c|c|c|}
\hline Young's Modulus [N/mm $\left.{ }^{2}\right]$ & Yield Stress [N/mm $\left.{ }^{2}\right]$ & Poisson's ratio \\
\hline $1.75 \mathrm{E} 05$ & 850 & 0.29 \\
\hline
\end{tabular}

Table 3: Material properties of the tool steel at $460 \mathrm{C}^{\circ}$

Boundary conditions. Since the numerical model has a large number of degrees of freedom, then the usage of contact boundary conditions increases its complexity. Therefore, the contact boundary conditions must be simplified to fully slip, stick or connecting degrees of freedom between the parts. Moreover, a special attention must be given in applying the right boundary conditions on the right surface; otherwise the model will be too stiff or too flexible. Concerning the billet, the following boundary conditions are applied as shown in Fig.13:

(1) Stick at cylinder-billet contact zone and die-billet contact zone

(2) Prescribed velocity of $5.3 \mathrm{~mm} / \mathrm{sec}$ at the inflow.

(3) Free in the extrusion direction at the outflow.

(4) A constraint equation is determined at each corner node between its displacement components in order to satisfy the material conservation condition [10].

Concerning the tool, the following boundary conditions are applied as shown in Fig.14:

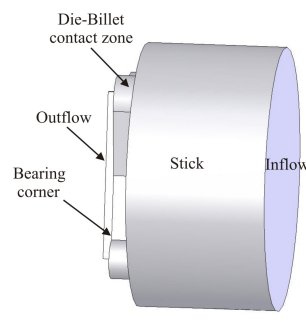

Fig.13: billet's boundaries

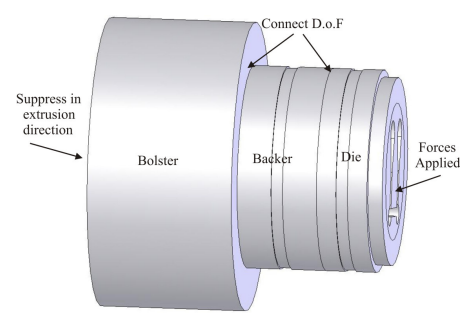

Fig.14: tool's boundaries 
(1) Suppress the downstream end of the bolster in the extrusion direction.

(2) The influence of the ring on the die deformation is neglected.

(3) Forces are applied at the nodes belonging to die-billet contact zone.

(4) The degrees of freedom of the contact nodes in the tool parts are connected in the following manner: nodes in the Bolster-Backer contact zone are connected in extrusion direction only, nodes in the Backer-Die contact zone are connected in three possibilities: in extrusion direction only, in all directions and in all directions except the tongue in the extrusion direction. Finally, the results from the three possibilities are compared.

Finite element models. The billet and the tool are discretized by a 10 node tetrahedron element. Each node has three translational degrees of freedom. They are discretized simultaneously to produce node-to-node contact. But the important point is the choice of how many elements per profile thickness to give sufficient results. Three meshes are checked with 2, 3 and 4 elements per profile thickness. Moreover, the use of more elements is limited with the solver or the computer capacity.

Numerical results. The angular deflection of the tongue of the mesh with 2 elements per profile thickness with three connection possibilities is summarized in table 4. In comparison with the experimental results, it is deduced that the last connection possibility is the most realistic one. In addition, Fig.15 confirms that only slip will occur between the die and the backer at the tongue. Regarding the three meshes, the results summarized in table 5 shows that three elements per profile thickness are sufficient to give accurate results. The three meshes are compared in number of degrees of freedom and not in calculation time because different solvers, direct and iterative are applied in these simulations. Finally, the deflection of the tool in the extrusion direction is exhibited in Fig.16. The relative linear displacement between points $\mathrm{A}$ and $\mathrm{B}$ in the extrusion direction is about $0.4 \mathrm{~mm}$ where the bearing length is $3 \mathrm{~mm}$.

\begin{tabular}{|l|c|}
\hline Connection possibility of D.o.F & Angular deflection of tongue [mrad] \\
\hline In extrusion direction & 10 \\
\hline In all directions & 5.25 \\
\hline In all directions except tongue in extrusion direction & 7 \\
\hline
\end{tabular}

Table 4: angular deflection of the tongue of the three connection possibilities

\begin{tabular}{|l|c|c|c|}
\hline Elements per thickness & Number of D.o.F & Extrusion force [MN] & $\begin{array}{c}\text { Angular deflection of } \\
\text { tongue [mrad] }\end{array}$ \\
\hline Two elements & 40131 & 1.03 & 7 \\
\hline Three elements & 104031 & 0.96 & 6.0 \\
\hline Four elements & 175914 & 0.96 & 6.0 \\
\hline
\end{tabular}

Table 5: number of degrees of freedom, extrusion force and angular deflection of the tongue for the finite element models

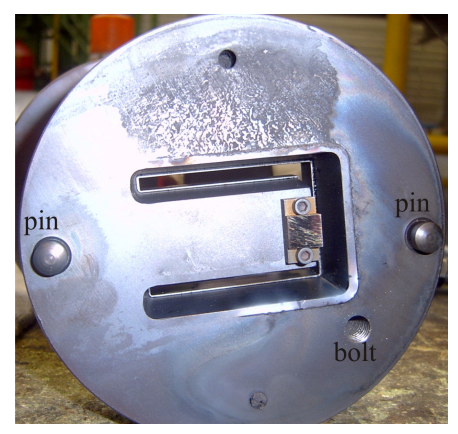

Fig.15: back side of the die

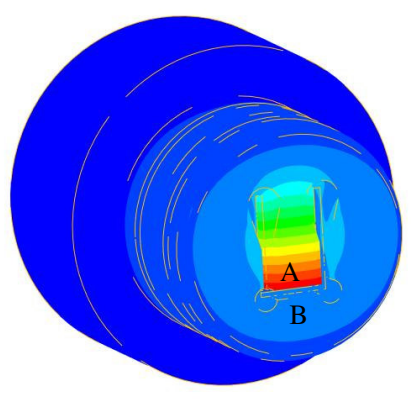

Fig.16: deflection of the tool in the extrusion direction [mm] 


\section{Conclusion}

Finally, it is concluded that the cheap and simple experiment succeeded in measuring the deformation of a flat die. It emphasizes that the die deforms elastically during the extrusion of the successors of the first billet while the origin of the plastic deformation that is found has not been yet clarified because the die is not tested directly after production. Concerning the numerical analysis, underestimated extrusion force and the angular deflection of the tongue are found in the comparison with the experimental results. The reason of the difference comes from the assumptions of aluminum material constants and isothermal analysis.

\section{Acknowledgement}

This research was carried out under project number MC4.05221A in the framework of the Material innovation institute research program in the Netherlands.

\section{References}

[1] T. Mori, N. Takatsuji, K.Matsuki, T.Aida, K.Murotani, K.Uetoko: Measurement of pressure distribution on die surface and deformation of extrusion die in hot extrusion of 1050 aluminum rod, Journal of Materials Processing Technology (2002), p421-425.

[2] B. Bourqui, A. Huber, C.Moulin, A.Brunetti, Y.Krähenbühl: Improved weld seam quality using 3D FEM simulation in correlation with practice (The First EAA Extruders Division Congress, Brescia 2002).

[3] P.T.Moe, S.Støren: A techniques for measuring pressure on the die face during extrusion ( Esaform, Kraków, 2002).

[4] M.Lefstad, P.T.Moe, S.Støren, R.Flatval: Thin strip aluminum extrusion - pressure, temperature and deflection recordings of the extrusion die (Esaform, Kraków, 2002).

[5] P.T.Moe: Pressure and Strain Measurement During Hot Extrusion of Aluminum (2005), p106112.

[6] T. Sheppard: Extrusion of Aluminum Alloys, Kluwer Academic Publishers (1999).

[7] W. Assaad, H.J.M. Geijselaers, J.Huétink: 3-D numerical simulation of direct aluminum extrusion and die deformation (Extrusion Technology, Orlando 2008).

[8] J.Lof: Developments in finite element simulations of aluminum extrusion (2000), p. 86

[9] H. Mooi: Finite element simulations of aluminum extrusion (1996), p. 127

[10] W. Assaad, H.J.M. Geijselaers, J.Huétink: Boundary conditions applied on bearing corner in direct aluminum extrusion (Esaform, Enschede 2009). 